\title{
Is age a risk factor for Candida glabrata colonisation?
}

\author{
Anurag N. Malani, ${ }^{1,2}$ Georgios Psarros, ${ }^{1}$ Preeti N. Malani ${ }^{1,3}$ and Carol A. Kauffman ${ }^{1}$ \\ ${ }^{1}$ Division of Infectious Diseases, Veterans Affairs Ann Arbor Healthcare System and University of Michigan Health System, Ann Arbor, MI, USA, ${ }^{2}$ Saint Joseph \\ Mercy Health System, Ann Arbor, MI, USA and ${ }^{3}$ Division of Geriatric Medicine and Geriatric Research Education and Clinical Center, Veterans Affairs Ann Arbor \\ Healthcare System and University of Michigan Health System, Ann Arbor, MI, USA
}

\section{Summary}

\begin{abstract}
Studies have reported that Candida glabrata infections are more common in older adults. We sought to determine colonisation rates of C. glabrata in the oral cavity and its relationship with age, comorbid illnesses and hospital or extended care facility stay. Samples were obtained from four sites in the oral cavity and from dentures, when available, from 408 subjects from the community (136), hospital (126) or an extended care facility (146). Overall, $219(53.7 \%)$ subjects were colonised with yeast; the predominant species was Candida albicans. Sixty-two patients (15.2\%) were colonised with C. glabrata. None of the subjects $<40$ years was colonised with $C$. glabrata; in those from the community, only nine persons, all of whom were $>60$ years, were colonised with C. glabrata. By multivariate analysis, increasing age, dentures and use of psychotropic medications were independently associated with C. glabrata colonisation; residing in the community, rather than hospital or extended care, was strongly protective against colonisation. Candida glabrata colonisation is multifactorial; age, and hospitalisation/extended care stay contribute to colonisation. Dentures are strongly associated with colonisation with any yeast and with C. glabrata. Further study is needed to evaluate the relationship of these findings to increasing C. glabrata infections in older adults.
\end{abstract}

Key words: Candida glabrata, colonisation, oral cavity.

\section{Introduction}

Candida species are an increasingly common cause of bloodstream infections in hospitalised patients. In some institutions, a shift in the species of Candida causing fungaemia has occurred with Candida glabrata being the most common non-albicans Candida species nowadays. ${ }^{1-5}$ As many as one quarter to one-third of all cases of fungaemia in some medical centres are caused by this species that is intrinsically more resistant to commonly used triazole agents.

The increase in the incidence of $C$. glabrata fungaemia appears to be multifactorial, and include increased use

Correspondence: C. A. Kauffman, MD, VA Healthcare System, 2115 Fuller Road, Ann Arbor, Ml 48105, USA.

Tel.: +1 (734) 845 3460. Fax: +1 (734) 8453290.

E-mail: ckauff@umich.edu

Accepted for publication 23 June 2010 of fluconazole, especially in haematology and stem cell transplant units, and geographical differences. ${ }^{2,4,5} \mathrm{Sev}-$ eral studies have reported that $C$. glabrata infections are more common in older adults than in younger individuals $^{2,6,7}$ and are uncommonly found in neonates and young children. ${ }^{3,6}$

The association between $C$. glabrata fungaemia and older age is poorly understood. One study that enrolled a small number of subjects found higher rates of $C$. glabrata oropharyngeal colonisation in adults over 80 years of age, ${ }^{8}$ but others have found no oropharyngeal colonisation with C. glabrata in older adults. ${ }^{9}$ A prior study from our institution noted that C. glabrata was a common coloniser among older residents of an extended care facility. ${ }^{10}$ We sought to extend these observations to a large sample of adults of various ages and to explore the association of age, comorbid illnesses and hospital or extended care facility stay with $C$. glabrata colonisation of the oral cavity. 


\section{Methods}

\section{Patients and setting}

Three populations of adults were studied from June 2006 to April 2008. The first group included healthy adults living in the community. The second group comprised adults who were hospitalised for at least 3 days in either medical or surgical units at the Veterans Affairs Ann Arbor Healthcare System or the St. Joseph Mercy Health System, Ann Arbor, Michigan. The third group comprised residents of extended care facilities in southeastern Michigan and included those in community nursing homes, the extended care unit at the Veterans Affairs Hospital and the long-term acute care unit at St. Joseph Mercy Health System. An attempt was made to enrol similar numbers of subjects of four age ranges, $18-39,40-59,60-79$ and $\geq 80$ years of age, in each of the above three categories.

This project was approved by the Institutional Review Boards of the University of Michigan, the Veterans Affairs Healthcare System and the St. Joseph Mercy Health System. Written informed consent was obtained from all subjects. If a subject in an extended care facility was unable to provide informed consent because of cognitive difficulties, written informed consent was obtained from their durable power of attorney.

Baseline demographic data and information related to risk factors were recorded. Information of interest included underlying medical conditions, denture use, prior surgical and dental procedures, and receiving immunosuppressive therapy, antibacterial agents and psychotropic drugs. Patients who had a diagnosis of thrush or who had received antifungal agents during the prior 30 days were excluded.

\section{Microbiological methods}

Samples were obtained from the buccal mucosa, posterior pharynx, palate, dorsal surface of the tongue and if applicable, upper dentures using individual sterile rayon-tipped applicator sticks. It was felt to be too difficult for some patients and too risky for others to do an oral rinse to obtain material for culture. Samples were streaked using a four-quadrant method onto BBL CHROMagar Candida (BD Diagnostics, Baltimore, MD, USA). Plates were incubated at $35^{\circ} \mathrm{C}$ for $72 \mathrm{~h}$. All phenotypically different cream, pink, purple or blue colonies were isolated for further study, and identification of C. glabrata was verified by the API 20C System (Bio-Merieux Vitek, Hazelwood, MO, USA). All phenotypically different green colonies were identified as
Candida albicans. The identification of other species by other colours was established by the API 20C System. The amount of growth on each plate was quantified as $1+(<10$ total colonies $), 2+(10-100$ total colonies $)$ and $3+(>100$ colonies). Colonisation was defined as at least one oral cavity site positive for Candida species.

\section{Data analysis}

Univariate analysis using simple logistic regression identified risk factors for yeast colonisation among study patients. A two-tailed P-value of 0.05 or less was considered statistically significant. Crude odds ratios (OR) and 95\% confidence intervals (CI) were calculated. Age was represented in a continuous fashion in our model with 1 year serving as the unit of measure. Variables that were significant at a $P$-value of 0.20 by univariate analysis, as well as those variables that had a priori clinical significance, were analysed using multivariable logistic regression modelling. All statistical analyses were performed using SAS 9.1 (SAS Institute Inc, Cary, NC, USA).

\section{Results}

The number of persons enrolled was 408, which included 136 (33.3\%) who lived in the community, $126(30.9 \%)$ who were residents of extended care facilities and $146(35.8 \%)$ who were hospitalised. Of the 408 subjects, $70(17.1 \%)$ were aged $18-39$ years, $113(27.7 \%)$ were aged 40-59 years, 149 (36.6\%) were aged $60-79$ years and $76(18.6 \%)$ were aged $>80$ years. A history of denture use was obtained in 124 persons, $102(82.2 \%)$ of whom were $>60$ years old.

\section{Yeast colonisation}

Oropharyngeal colonisation with any yeast species was found in 219 subjects (53.7\%). Colonisation was much less common in those $<40$ years of age when compared with the older age groups. Only 15 (21.4\%) subjects aged 18-39 years were colonised with any yeast species at any site, whereas $54.9 \%$ of those between the ages 40 and 59 years, $63.1 \%$ of those aged $60-79$ years and $63.2 \%$ of those aged older than 80 years were colonised, OR, 1.03 (95\% CI, 1.02-1.04) (Table 1). The OR of 1.03 suggests that for every decade, the odds of having yeast colonisation increases by $30 \%$. We did not observe a difference in colonisation rates by gender $(P=0.52)$. Of the 219 persons colonised, $43(19.6 \%)$ were from the community, 87 (39.7\%) from extended care facilities and 89 (40.6\%) from hospitals. When compared with 
Table 1 Characteristics of subjects with colonisation with any yeast compared with those without colonisation.

\begin{tabular}{lcccc}
\hline & $\begin{array}{c}\text { Colonised } \\
\text { with yeast }\end{array}$ & $\begin{array}{c}\text { Not colonised } \\
\text { with yeast }\end{array}$ & OR $(95 \% \text { Cl) })^{1}$ & P-value \\
\hline Number & 219 & 189 & & \\
Age $^{2}$ & $65.8 \pm 16.1$ & $54.7 \pm 21.9$ & $1.03(1.02-1.04)$ & $<0.001$ \\
Location & $43(19.6)$ & $93(49.2)$ & $0.25(0.16-0.39)$ & $<0.001$ \\
$\quad$ Community & $87(39.7)$ & $39(20.6)$ & & \\
$\quad$ Extended care & $89(40.6)$ & $57(30.2)$ & & \\
$\quad$ Hospital & $141(64.3)$ & $118(62.4)$ & $1.1(0.7-1.6)$ & 0.68 \\
Males & $69(31.5)$ & $39(20.6)$ & $1.8(1.1-2.8)$ & 0.01 \\
Diabetes & $4(2)$ & $5(3)$ & $0.7(0.2-2.6)$ & 0.57 \\
Radiation therapy & $4(2)$ & $5(3)$ & $0.7(0.2-2.6)$ & 0.57 \\
Head/neck cancer & $56(25.6)$ & $20(10.6)$ & $2.9(1.7-5.1)$ & $<0.001$ \\
COPD & $21(9.6)$ & $25(13.2)$ & $0.7(0.4-1.3)$ & 0.25 \\
Asthma & $72(32.9)$ & $28(14.9)$ & $2.8(1.7-4.6)$ & $<0.001$ \\
Dry mouth & $9(4.1)$ & $3(1.6)$ & $2.7(0.7-10)$ & 0.13 \\
Cirrhosis & $96(43.8)$ & $28(14.8)$ & $4.9(2.7-7.2)$ & $<0.001$ \\
Dentures & $45(20.6)$ & $22(11.7)$ & $2.0(1.1-3.4)$ & 0.02 \\
Periodontal disease & $34(15.5)$ & $14(7.4)$ & $2.3(1.2-4.4)$ & 0.01 \\
Steroid inhaler & $41(18.7)$ & $14(7.4)$ & $2.9(1.5-5.4)$ & $<0.001$ \\
Immunosuppressives & $102(46.6)$ & $48(25.4)$ & $2.5(1.7-3.9)$ & $<0.001$ \\
Psychotropic agents & $117(53.4 .6)$ & $60(31.8)$ & $2.4(1.6-3.7)$ & $<0.001$ \\
Antibiotics & $82(37.4)$ & $43(22.8)$ & $2.0(1.3-3.1)$ & 0.001 \\
Recent hospitalisation ${ }^{3}$ & & & & \\
& & & &
\end{tabular}

COPD, chronic obstructive pulmonary disease.

${ }^{1}$ Odds ratio and $95 \%$ confidence interval.

${ }^{2} \mathrm{OR}$ for age reported for 1-year unit.

${ }^{3}$ Hospitalisation within the previous 30 days. patients from extended care facilities and hospitals, subjects from the community had only $25 \%$ as much risk of colonisation [OR: 0.25 (CI: 0.16-0.39), $P<0.001]$.

Yeast colonisation was associated with many factors by univariate analysis (Table 1). A strong association was found with dentures. A total of 124 patients wore dentures; in 99 patients, these were upper dentures. By multivariate analysis, the independent factors associated with colonisation were age, denture use and receipt of psychotropic agents (Table 2).

The most common colonising species was C. albicans, found in 192 (88\%) subjects, followed by C. glabrata in $62(28 \%)$ and Candida tropicalis in 11 (5\%) subjects. Other species including Candida parapsilosis, Candida famata, Candida lusitaniae and Saccharomyces cerevisiae were uncommonly found. A total of 55 (25.1\%) persons were colonised with two species, and 44 of these 55 were colonised with C. glabrata and C. albicans. Among denture wearers, C. albicans was the most common species isolated, followed by C. glabrata. Of the 99 upper dentures, 84 were available for cultures to be taken and $59(70.2 \%)$ samples resulted in the growth of yeast. Of the other sites, $187 / 407(45.9 \%)$ of the dorsal tongue samples, $172 / 406(42.4 \%)$ of oropharyngeal samples, $160 / 408(39.2 \%)$ of buccal samples and $147 / 397$
Table 2 Multivariate analysis of select patient characteristics associated with colonisation by any yeast.

\begin{tabular}{|c|c|c|c|}
\hline & $\begin{array}{l}\text { Unadjusted } \\
\text { OR }(95 \% \mathrm{Cl})^{1}\end{array}$ & $\begin{array}{l}\text { Adjusted } \\
\text { OR }(95 \% \mathrm{Cl})^{1}\end{array}$ & $P$-value \\
\hline $\mathrm{Age}^{2}$ & $1.03(1.02-1.04)$ & $1.02(1.0-1.05)$ & 0.01 \\
\hline Dentures & $4.9(2.7-7.2)$ & $3.5(2.1-6.1)$ & $<0.001$ \\
\hline Psychotropic agents & $2.5(1.7-3.9)$ & $1.8(1.1-2.9)$ & 0.02 \\
\hline Presence of dry mouth & $2.8(1.7-4.6)$ & $1.6(0.9-2.7)$ & 0.11 \\
\hline Receipt of antibiotics & $2.4(1.6-3.7)$ & $1.6(0.9-2.6)$ & 0.08 \\
\hline Recent hospitalisation ${ }^{3}$ & $2.0(1.3-3.1)$ & $1.5(0.9-2.6)$ & 0.10 \\
\hline
\end{tabular}

${ }^{1}$ Odds ratios and $95 \%$ confidence intervals based on a multivariable logistic regression adjusting for age, dentures, use of psychotropic agents, presence of dry mouth, receipt of antibiotics and recent hospitalisation.

${ }^{2}$ OR for age reported for 1-year unit.

${ }^{3}$ Hospitalisation within the previous 30 days.

(37\%) of palate samples resulted in the growth of yeast (Fig. 1).

\section{Candida glabrata colonisation}

Colonisation with C. glabrata was found in 62 subjects (15.2\%), including 15 (13.2\%) of those aged 4059 years, $31(20.8 \%)$ of those aged 60-79 years and 


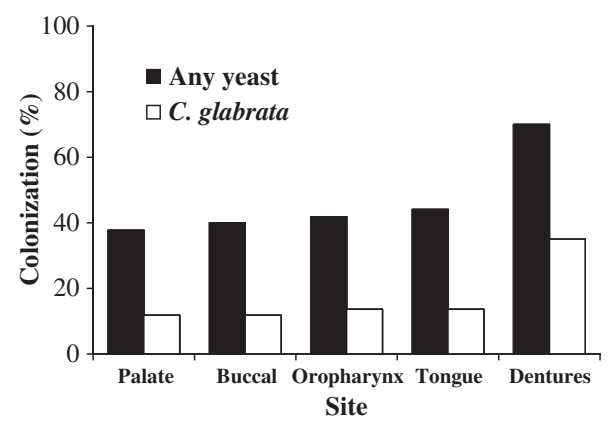

Figure 1 Oral cavity colonisation with any yeast and with Candida glabrata in relation to site.

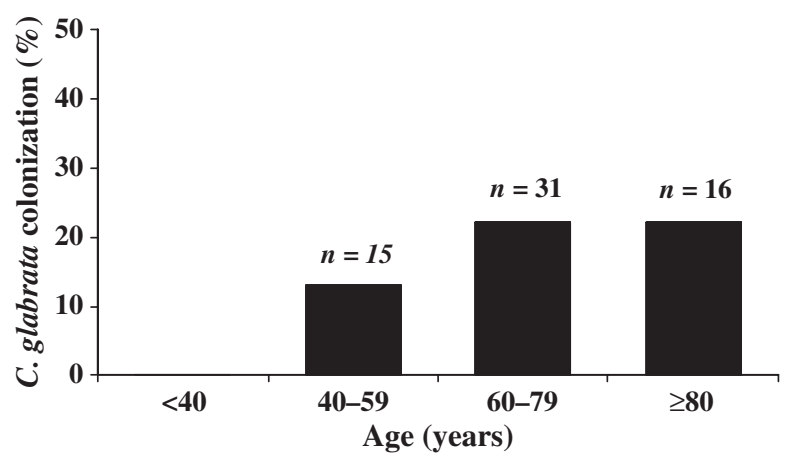

Figure 2 Oral cavity colonisation with Candida glabrata in relation to age.

$16(21.1 \%)$ of those aged $\geq 80$ years of age (Fig. 2$)$. No person $<40$ years of age was colonised with C. glabrata. Only $9 / 136(6.6 \%)$ of persons from the community, all of whom were $>60$ years of age, were colonised with $C$. glabrata, compared with $28 / 126(22.2 \%)$ residents of extended care facilities and 25/146 (17.1\%) hospitalised patients. Of the nine persons who lived in the community and who had C. glabrata colonisation, eight wore dentures. The mean age of persons colonised with C. glabrata was $71.5 \pm 12.4$ years, compared with those not colonised with $C$. glabrata, $58.7 \pm 20.2$ years $(P<0.001)$ and those not colonised with any yeast, $54.6 \pm 21.9$ years $(P<0.001)$. Colonisation with $C$. glabrata varied among different sites in the oral cavity, but generally was similar to that noted for any yeast; $33.3 \%$ of dentures yielded C. glabrata when sampled (Fig. 1). The amount of growth of C. glabrata varied among different locations in the mouth and in different age groups. Dentures were the site most heavily colonised, and subjects $>60$ years of age had heavier growth of yeast from all sites compared with younger subjects (data not shown).
Univariate analysis of risk factors showed that age, diabetes mellitus, denture use and use of psychotropic agents were associated with colonisation with C. glabrata, and living in the community was protective against colonisation (Table 3). Multivariate analysis showed that increasing age, dentures and use of psychotropic medications were independently associated with $C$. glabrata colonisation, and community dwelling was strongly protective (Table 4).

\section{Discussion}

Colonisation of the oral cavity with Candida species has been studied in different patient populations, including patients with HIV infection, ${ }^{11,12}$ those with diabetes, ${ }^{13}$ neonates, children, older adults ${ }^{8,9,14,15}$ and residents of extended care facilities. ${ }^{10,16,17}$ In all these studies, $C$. albicans has been the yeast most commonly isolated from the oral cavity. Few studies have focused on colonisation of the oral cavity with C. glabrata.

We found that C. glabrata colonisation of the oral cavity increased with age, and this increase appeared to be independent of denture use. The effect of increasing age on C. glabrata colonisation was previously noted in a study on 93 community-dwelling older adults, aged 60 to $>80$ years. $^{8}$ In that study, comparison with a younger age group was not performed. The results differed from the present study in that $C$. glabrata was found only in those $>70$ years of age, and among persons without dentures, C. glabrata was found only in those $>80$ years of age. ${ }^{8}$ Another study of oral yeast colonisation that compared 239 healthy subjects in China with 483 healthy subjects in North America between the ages of 15 and 76 years, found only one isolate of C. glabrata in each population group. $^{16}$ However, the samples were taken from the gingival mucosa, which may not reflect the areas more likely to demonstrate colonisation by C. glabrata.

One could argue that our findings may have differed if we had used oral rinses rather than swabbing to isolate yeast from the oral cavity. It is possible that we missed low-level colonisation with yeast by using swabs instead of rinses. However, we wished to identify colonisation at different sites within the oral cavity and that is not feasible with the oral rinse technique. In addition, it is difficult for hospitalised patients and some nursing home patients to carry out the rinse procedure, and this could have led to unintended differences among the groups and thus skewed the results.

In addition to older age, psychotropic drugs and dentures emerged as important risk factors for C. glabrata oral colonisation. Xerostomia is a frequent side effect of 
Table 3 Characteristics of subjects with Candida glabrata colonisation compared with those without $C$. glabrata colonisation.

\begin{tabular}{lcccc}
\hline & $\begin{array}{l}\text { Colonised with } \\
\text { C. glabrata }\end{array}$ & $\begin{array}{l}\text { Not colonised with } \\
\text { C. glabrata }\end{array}$ & OR (95\% Cl) & P-value \\
\hline Number & 62 & 346 & & \\
Age & $71.5 \pm 12.4$ & $58.7 \pm 20.2$ & $1.04(1.02-1.06)$ & $<0.001$ \\
Location & & & & \\
$\quad$ Community & $9(14.5)$ & $127(36.7)$ & $0.29(0.14-0.61)$ & $<0.001$ \\
$\quad$ Extended care & $28(45.2)$ & $98(28.3)$ & & \\
$\quad$ Hospital & $25(40.3)$ & $121(35)$ & & \\
Males & $35(56)$ & $224(65)$ & $0.8(0.4-1.3)$ & 0.36 \\
Diabetes & $24(38.7)$ & $84(24.3)$ & $2.0(1.1-3.5)$ & 0.02 \\
Radiation therapy & $2(3.2)$ & $7(2)$ & $1.6(0.3-8)$ & 0.63 \\
Head/neck cancer & $2(3.2)$ & $7(2)$ & $1.6(0.3-8)$ & 0.63 \\
COPD & $15(24.2)$ & $61(17.6)$ & $1.5(0.8-2.8)$ & 0.22 \\
Asthma & $7(11.3)$ & $39(11.3)$ & $1.0(0.4-2.4)$ & 1.0 \\
Dry mouth & $21(34)$ & $79(23)$ & $1.7(0.96-3.1)$ & 0.06 \\
Cirrhosis & $4(6.5)$ & $8(2.3)$ & $2.9(0.85-10)$ & 0.09 \\
Dentures & $45(72.6)$ & $79(22.8)$ & $8.9(4.9-16.5)$ & $<0.001$ \\
Periodontal disease & $15(24.2)$ & $52(15)$ & $1.8(0.94-3.5)$ & 0.07 \\
Steroid inhaler & $10(16.1)$ & $38(11)$ & $1.6(0.73-3.3)$ & 0.25 \\
Immunosuppressives & $11(17.7)$ & $44(12.8)$ & $1.5(0.7-3)$ & 0.29 \\
Psychotropic agents & $33(53.2)$ & $117(33.8)$ & $2.2(1.3-3.8)$ & 0.004 \\
Antibiotics & $32(51.6)$ & $145(41.9)$ & $1.5(0.9-2.5)$ & 0.16 \\
Recent hospitalisation ${ }^{3}$ & $23(37.1)$ & $102(29.5)$ & $1.4(0.8-2.5)$ & 0.23 \\
\hline
\end{tabular}

COPD, chronic obstructive pulmonary disease.

${ }^{1}$ Odds ratio and $95 \%$ confidence interval.

${ }^{2} \mathrm{OR}$ for age reported for 1-year unit.

${ }^{3}$ Hospitalisation within the previous 30 days.
Table 4 Multivariate analysis of select patient characteristics associated with Candida glabrata colonisation.

\begin{tabular}{lllc}
\hline & Unadjusted & Adjusted & \\
& OR $(95 \% \mathrm{Cl})^{1}$ & OR $(95 \% \mathrm{Cl})^{1}$ & $P$-value \\
\hline Age $^{2}$ & $1.04(1.02-1.06)$ & $1.01(1.001-1.03)$ & 0.03 \\
Diabetes & $2.0(1.1-3.5)$ & $1.1(0.65-1.8)$ & 0.76 \\
Dentures & $8.9(4.9-16.5)$ & $3.1(1.8-5.3)$ & $<0.001$ \\
Psychotropic agents & $2.2(1.3-3.8)$ & $1.7(1.01-2.7)$ & 0.05 \\
Community dwelling & $0.29(0.14-0.61)$ & $0.45(0.26-0.77)$ & 0.004 \\
\hline
\end{tabular}

${ }^{1}$ Odds ratios and $95 \%$ confidence intervals based on a multivariable logistic regression adjusting for age, diabetes, dentures, use of psychotropic agents and patient location (community dwelling or not).

${ }^{2}$ OR for age reported for 1-year unit.

certain psychotropic drugs and may predispose to oral candidiasis. ${ }^{18,19}$ Low salivary flow rates were noted to be inversely related to the intensity of saliva carriage with $C$. albicans and $C$. parapsilosis, but were not studied in relation to $C$. glabrata. ${ }^{19}$ However, we found no significant association with xerostomia although our definition was based on the patient's perception of whether they had dry mouth and not on an objective measurement of salivary flow.

Denture use is known to be a risk factor for oral cavity yeast colonisation ${ }^{20-22}$ and also has been linked to
C. glabrata oral colonisation in one study. ${ }^{8}$ In our study, denture wearers were three times more likely to harbour C. glabrata than those who wore no dentures, and this emerged as the strongest risk factor for colonisation. Most experimental work on the adherence of Candida to denture materials has used $C$. albicans as the test organism because it is the predominant yeast found colonising dentures. Only a few studies have been performed with C. glabrata. These have shown that this species can adhere to both cold-cured and heat-cured acrylic resins that are commonly used to manufacture dentures and that adherence to dental acrylic of many, but not all, C. glabrata strains was greater than that of C. albicans strains. ${ }^{23,24}$

An important finding from our study was that C. glabrata colonisation was uncommon in community-dwelling persons, regardless of age, but was much more common in those in hospital or residing in an extended care facility. There was no difference of the rates of colonisation between persons in hospital and those in an extended care facility. There were no community-dwelling subjects $<60$ years of age who had C. glabrata colonisation. This raises the question of whether $C$. glabrata is acquired in the hospital or extended care setting or is perhaps selected for in those settings. We did not find $C$. glabrata to be a major 
coloniser of hands of health care workers in a prospective study in an intensive care unit, nor did we find evidence of transmission to patients in this setting. ${ }^{25}$ Others have described nosocomial spread of C. glabrata and have found this species contaminating environmental surfaces, but not the hands of health care workers. $^{26}$

The results from this study should not be interpreted as providing evidence that another agent, other than fluconazole, should be used to treat thrush or denture stomatitis in older adults. The subjects in this study did not have thrush and were asymptomatically colonised with yeast. This study was not aimed at addressing the issue of defining the aetiological agent causing thrush in these different populations.

Whether increased oral colonisation with C. glabrata in older adults, especially those in hospital and extended care settings, has relevance to the increase in bloodstream infections caused by this organism in older adults is not addressed in this study. It is highly unlikely that the oral cavity is a site from which dissemination occurs. Dissemination more likely originates from the bowel, and assessment of growth in stool or rectal colonisation with $C$. glabrata would be more directly relevant to this question. Unfortunately, it is difficult to enlist community-dwelling individuals to undergo swabbing of the rectum or provide a stool specimen, and we thought it was important in this study to compare community-dwelling persons with those in hospital and extended care facilities. Indirect evidence was obtained in our prior study in the intensive care setting, in which both oral and rectal colonisation was assessed. Most patients were found to have the same yeast species at both locations, and C. glabrata was the most common coloniser of the rectum in patients in the surgical intensive care unit. ${ }^{25}$ Only when studies are performed that correlate specific strains causing fungaemia with those colonising various body sites, including the oral cavity, will the role of increased colonisation with $C$. glabrata with increasing age, be better defined.

\section{Conflicts of interest}

None of the authors has any conflicts of interest to report.

\section{References}

1 Baddley JW, Smith AM, Moser SA, Pappas PG. Trends in frequency and susceptibilities of Candida glabrata bloodstream isolates at a university hospital. Diagn Microbiol Infect Dis 2001; 39: 199-201.
2 Malani PN, Bradley SF, Little RS, Kauffman CA. Trends in species causing fungaemia in a tertiary care medical centre over 12 years. Mycoses 2001; 44: 446-9.

3 Pappas PG, Rex JH, Lee J et al. A prospective observational study of candidemia: epidemiology, therapy, and influences on mortality in hospitalized adult and pediatric patients. Clin Infect Dis 2003; 37: 634-43.

4 Rangel-Frausto MS, Wiblin T, Blumberg HM et al. National epidemiology of mycoses survey (NEMIS): variations in rates of bloodstream infections due to Candida species in seven surgical intensive care units and six neonatal intensive care units. Clin Infect Dis 1999; 29: 253-8.

5 Marr KA, Seidel K, White TC, Bowden RA. Candidemia in allogeneic blood and marrow transplant recipients: evolution of risk factors after the adoption of prophylactic fluconazole. J Infect Dis 2000; 181: 309-16.

6 Malani A, Hmoud J, Chiu L, Carver PL, Bielaczyc A, Kauffman CA. Candida glabrata fungemia: experience in a tertiary care center. Clin Infect Dis 2005; 41: 975-81.

7 Diekema DJ, Messer SA, Brueggemann AB et al. Epidemiology of candidemia: 3-year results from the Emerging Infections and the Epidemiology of Iowa Organisms Study. J Clin Microbiol 2002; 40: 1298-302.

8 Lockhart SR, Joly S, Vargas K, Swails-Wenger J, Enger L, Soll DR. Natural defenses against Candida colonization breakdown in the oral cavities of the elderly. J Dent Res 1999; 78: 857-68.

9 Kleinegger CL, Lockhart SR, Vargas K, Soll DR. Frequency, intensity, species, and strains of oral Candida vary as a function of host age. J Clin Microbiol 1996; 34: 2246-54.

10 Hedderwick SA, Wan JY, Bradley SF, Sangeorzan JA, Terpenning MS, Kauffman CA. Risk factors for colonization with yeast species in a Veterans Affairs long-term care facility. J Am Geriatr Soc 1998; 46: 849-53.

11 Sangeorzan JA, Bradley SF, He X et al. Epidemiology of oral candidiasis in HIV-infected patients: colonization, infection, treatment, and emergence of fluconazole resistance. Am J Med 1994; 97: 339-46.

12 Sanchez-Vargas LO, Ortiz-Lopez NG, Villar M et al. Point prevalence, microbiology and antifungal susceptibility patterns of oral Candida isolates colonizing or infecting Mexican HIV/AIDS patients and healthy persons. Rev Iberoam Micol 2005; 22: 83-92.

13 Goncalves RH, Miranda ET, Zaia JE, Giannini MJ. Species diversity of yeast in oral colonization of insulin-treated diabetes mellitus patients. Mycopathologia 2006; 162 : 83-89.

14 Qi QG, Hu T, Zhou XD. Frequency, species and molecular characterization of oral Candida in hosts of different age in China. J Oral Pathol Med 2005; 34: 352-6.

$15 \mathrm{Xu}$ J, Mitchell TG. Geographical differences in human oral yeast flora. Clin Infect Dis 2003; 36: 221-4.

16 Grimoud AM, Marty N, Bocquet H, Andrieu S, Lodter JP, Chabanon G. Colonization of the oral cavity by Candida 
species: risk factors in long-term geriatric care. J Oral Sci 2003; 45: 51-55.

17 Wilkieson C, Samaranayake LP, MacFarlane TW, Lamey P-J, MacKenzie D. Oral candidosis in the elderly in long term hospital care. J Oral Pathol Med 1991; 20: 13-16.

18 Astor FC, Hanft KL, Ciocon JO. Xerostomia: a prevalent condition in the elderly. Ear Nose Throat J 1999; 78: 476-9.

19 Torres SR, Peixoto CB, Caldas DM et al. Clinical aspects of Candida species carriage in saliva of xerotomic subjects. Med Mycol 2003; 41: 411-5.

20 Berdicevsky I, Ben-Aryeh H, Szargel R, Gutman D. Oral candida of asymptomatic denture wearers. Int J Oral Surg 1980; 9: 113-5.

21 Budtz-Jorgensen E, Stenderup A, Grabowski M. An epidemiologic study of yeasts in elderly denture wearers. Community Dent Oral Epidemiol 1975; 3: 115-9.
22 Cardash HS, Helft M, Shani A, Marshak B. Prevalence of Candida albicans in denture wearers in an Israeli geriatric hospital. Gerodontology 1989; 8: 101-7.

23 He XY, Meurman JH, Kari K, Rautemaa R, Samaranayake LP. In vitro adhesion of Candida species to denture base materials. Mycoses 2005; 49: 80-84.

24 Luo G, Samaramayake LP. Candida glabrata, an emerging fungal pathogen, exhibits superior relative cell surface hydrophobicity and adhesion to denture acrylic surfaces compared with Candida albicans. AAPMIS 2002; 110 : 601-10.

25 Hedderwick SA, Lyons MJ, Liu M, Vazquez JA, Kauffman CA. Epidemiology of yeast colonization in the intensive care unit. Eur J Clin Microbiol Infect Dis 2000; 19: 663-70.

26 Vazquez J, Dembry LM, Sanchez V et al. Nosocomial Candida glabrata colonization: an epidemiological study. J Clin Microbiol 1998; 36: 421-6. 